\title{
Measuring ocean waves in sea ice using SAR imagery: A quasi-deterministic approach evaluated with Sentinel-1 and in situ data
}

\author{
Ardhuin Fabrice ${ }^{1,{ }^{*}}$, Stopa Justin ${ }^{2,9}$, Chapron Bertrand ${ }^{9}$, Collard Fabrice ${ }^{3}$, Smith Madison ${ }^{4}$, \\ Thomson Jim ${ }^{4}$, Doble Martin ${ }^{5}$, Blomquist Byron ${ }^{6}$, Persson Ola ${ }^{6}$, Collins Clarence O. ${ }^{7}$, \\ Wadhams Peter $^{8}$
}

${ }^{1}$ Univ. Brest, CNRS, IRD, Ifremer, Laboratoire d'Océanographie Physique et Spatiale (LOPS), IUEM, Brest, France

${ }^{2}$ LHEEA, Ecole Centrale de Nantes, France

${ }^{3}$ OceanDataLab, Brest, France

${ }^{4}$ University of Washington, Seattle, USA

${ }^{5}$ PolarScientific Ltd., Appin, UK

${ }^{6}$ NOAA/Earth System Research Laboratory, CIRES, University of Colorado Boulder, Boulder, CO, USA

${ }^{7}$ Oceanography Division, Naval Research Laboratory, Stennis Space Center, Hancock County, MS, USA

${ }^{8}$ Cambridge Polar Consultants Ltd., Cambridge, UK

* Corresponding author : Fabrice Ardhuin

\begin{abstract}
:
Measurements of wave heights in marginal ice zones are limited to very few in situ data. Here we revisit the linear and quasilinear theories of Synthetic Aperture Radar imaging of waves in the particular case of waves in sea ice. Instead of only working with spectra, we have developed an iterative nonlinear algorithm to estimate phase-resolved deterministic maps of wave-induced orbital velocities, from which elevation spectra can be derived. Application of this algorithm to Sentinel $1 \mathrm{~A}$ wave mode images in the Southern Ocean shows that it produces reasonable results for swells in all directions except when they propagate at a few degrees off the range direction. The estimate of wave parameters is expected to work best when the shortest wave components, those which cause a pixel displacement of the order of the dominant wavelength in azimuth, can be neglected. Otherwise short waves produce a blurring of the image, increasing exponentially with the azimuthal wavenumber and reducing the estimated wave amplitude. Given the expected spatial attenuation of waves in ice-covered regions, our deterministic method should apply beyond a few tens of kilometers in the ice, without any correction for short wave effects. In situ data collected around the ice edge as part of the 2015 SeaState DRI cruise in the Beaufort confirm the progressive image blurring caused by such short waves, and the apparent reduction in the wave modulation. When short waves propagate from the open ocean towards the ice, this blurring can produce an unrealistic apparent increase of wave height, from the open ocean up to a few tens of kilometers inside the ice.
\end{abstract}




\section{Highlights}

- An algorithm is proposed to obtain orbital velocity maps from SAR images over sea ice. The algorithm is validated in terms of ocean wave spectra using in situ measurements. Wave height retrieval works best in the absence of unresolved short waves.

Keywords : SAR, Sentinel-1A, Ocean waves, Sea ice 


\section{Introduction}

A knowledge of wave parameters in sea ice is critical for navigation safety as well as for the understanding ocean currents and mixing near the ice (e.g. Rascle and Ardhuin, 2009) and the evolution of the ice edge (e.g. Davis et al., 2016). A quantitative estimation of wave height from satellite remote sensing would greatly augment the amount of available data in the marginal ice zones, where only few experiments have been performed (e.g. Wadhams et al., 1986; Kohout et al., 2014; Doble et al., 2015; Sutherland and Gascard, 2016). With their all-weather capabilities and unprecedented coverage, the high resolution modes of the Sentinel 1 constellation provides an extensive set of Synthetic Aperture Radar (SAR) images of the ice-covered ocean.

There have been many contributions to the quantitative analysis of waves in the open ocean using analyses of the image spectrum (e.g. Hasselmann and Hasselmann, 1991), and further adjustments in the spatial domain using the autocorrelation function (Collard et al., 2005). In ice-covered regions, patterns in SAR imagery due to ocean wave have also been reported by Lyzenga et al. (1985) among others. When such patterns are present, the dominant peak in the power spectrum of the image intensity yields the dominant wavelength and wave direction (e.g. Liu et al., 1991; Shulz-Stellenfleth and Lehner, 2002). Also, using the observed attenuation of waves and change of wavelength Wadhams et al. (2004) proposed to determine the ice thickness. Such an application, however, requires a good understanding of the relationship between wave heights and patterns in the SAR image. This understanding is the main topic of the present paper.

The apparent modulation of the radar backscatter is expected to be caused by the velocity bunching effect. Velocity bunching is a displacement along the direction of motion of the radar antenna (the azimuth) of the scatterers in the SAR image. This displacement is due to the fact that both the position in azimuth and the scatterer velocity towards the radar contribute to the Doppler shift received by the radar. Hence, a homogeneous roughness with a spatial variation of velocities creates patterns in the SAR scene. A more complete interpretation of SAR scenes in the ice-covered ocean was proposed by Vachon et al. (1993) who also included tilt effects. Given the generally weak large-scale variation of radar backscatter with incidence angles over the ice, weaker than over water, in this work we will neglect here the tilting of the icy surface.

Because the velocity bunching can result in a highly nonlinear transformation of wave patterns into an image, the spectrum of the image can have a very different shape from the surface elevation spectrum. Consequently, the image contrast is not a monotonic function of the wave amplitude (e.g. Liu et al., 1991), which complicates a quantitative analysis of wave properties from SAR imagery.

Lyzenga et al. (1985) also showed how the velocity bunching transformed straight lines, like the ice edge, into oscillating contours. That idea was extended by Ardhuin et al. (2015) to the analysis of wave patterns within the ice pack, and the estimation of wave heights in the presence of two swell systems.

The expected displacement of targets in the azimuth direction is given by their velocity multiplied by the ratio of distance $R$ to the satellite velocity along its orbit $V$. The target velocity can be decomposed into a vertical component $w$, and a horizontal component $u$, giving the dis- 
placement

$$
\delta_{A}=\left(w \cos \theta_{i}+u \sin \theta_{i}\right) R / V
$$

where $\theta_{i}$ is the incidence angle, and $Z=R \cos \theta_{i}$ is the satellite altitude above the target, with $R$ the radar-target distance. In ice-covered conditions, Ardhuin et al. (2015) further neglected the horizontal ice motion giving

$$
\delta_{A} \simeq w Z / V
$$

The in situ data from SWIFT drifters (Thomson, 2012), discussed in section 3, instead show that the vertical and horizontal motions have nearly equal amplitudes. In the particular event analyzed here, this applies within $4 \mathrm{~km}$ of the ice edge, and wave frequencies up to $0.2 \mathrm{~Hz}$. Those data were taken within $4 \mathrm{~km}$ of the ice edge, and in wave frequencies below $0.2 \mathrm{~Hz}$; it not clear how general this property may change with ice thickness and ice floe sizes. To include the $u$ component we define the velocity $v$ such that

$$
\delta_{A}=v Z / V=\left(w+u \tan \theta_{i}\right) Z / V .
$$

Because ocean waves are random, $v$ and $\delta_{A}$ are random. All wave components traveling in the azimuth direction and with a wavelength shorter than the typical value of $\delta_{A}$ are blurred by the random displacements (Alpers and Rufenach, 1979). This effect is known as the azimuthal cut-off (e.g. Kerbaol et al., 1998). Because it is a prominent feature of spectra of SAR images, the cut-off has also been used to measure the root mean square orbital velocity of surface gravity waves (Stopa et al., 2015).

In ice-covered water, the short waves are quickly attenuated by their interaction with the ice (e.g. Squire et al., 1995), so that the blurring
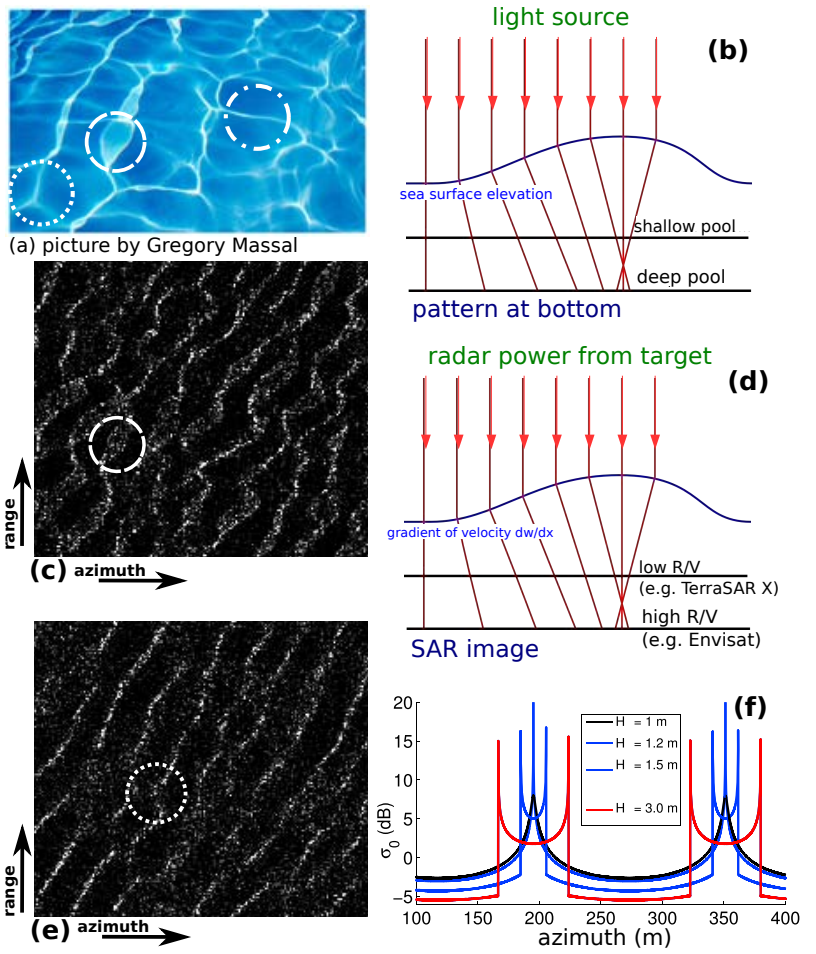

Figure 1: Analogy between (a) light patterns at the bottom of a shallow pool, and (c) and (e) velocity bunching effects along the azimuth direction in SAR images of ocean waves. (c) and (e) are taken from a wave mode Sentinel 1A image, acquired on 9 September 2014 at 04:48:16 UTC, at 10 and $35 \mathrm{~km}$ inside of the sea ice. In (c), almost all crests are doubled, for example in the region within the dashed circle. In (e) the lines are less bright and not doubled (for example within the dotted circle). This is easily simulated, as shown in (f), with the variations of image intensity expected from a sinusoidal monochromatic wave of wavelength $156 \mathrm{~m}$. An important difference is that there are also bright lines along the $x$-axis in the pool image (e.g. dash-dotted circle). 
is weak. Instead the pixel displacement $\delta_{A}$ enhances coherent long waves propagating in azimuth. In many cases there is a simple relationship between the modulation in the image intensity and the rate of change of the vertical velocity along the azimuth direction. As already described by Hasselmann et al. (1985) and illustrated here in Figure 1, the imaging of surface waves by the velocity bunching is exactly analogous to the mapping of the surface elevation by light patterns at the bottom of a pool. It is noteworthy that this optical technique has been used by Cox (1958) and Jähne and Riemer (1990). The main difference is that SAR imagery over the ice-covered ocean produces focusing only in the azimuth direction, and no such focusing in the range direction. Range bunching that is common in SAR images over land with mountain ranges correspond to cases when the ground or sea surface slope is comparable to the incidence angle.

The difference here is that the displacement in the image is not proportional to the surface slope but, as given by eq. (1), it is proportional to the surface velocity. As a result, the SAR image is brighter where $-\partial v / \partial y$ is maximum, with $y$ the azimuth direction.

Here we discuss how wave elevation spectra can be derived from various patterns in SAR images over sea ice using amplitude images. We particularly wish to take advantage of the sampling and coverage of the wave mode of the Sentinel-1 constellation. This wave mode extends the previous capabilities of ERS $1 / 2$ and Envisat, and is ideally designed to map the largescale evolution of wave properties across ocean basins (Collard et al., 2009; Hasselmann et al., 2012).

Our general objective is to define a level-2 processing to determine ocean wave spectra in ice- covered regions, as is currently done in open water (Chapron et al., 2001; Johnsen et al., 2006; Johnsen and Collard, 2009). A method is proposed in section 2 , using brightness patterns and spectral shapes. Following Hasselmann and Hasselmann (1991) our method uses the complete nonlinear SAR transformation, because nonlinear features are very common in SAR scenes over sea ice and the quasi-linear approach of Chapron et al. (2001) is not sufficient. Because short wind seas can often be neglected, our method is particularly adapted to the narrow wave spectra generally found in sea ice. The same method applies to both wave mode and interferometric wide (IW) swath images from Sentinel-1, as illustrated in section 3 . With that example we also use in situ wave measurements acquired near the ice edge during a 2015 field experiment in the Beaufort Sea (Wadhams and Thomson, 2015), in order to evaluate the wave estimates from our method, including the effect of short unresolved waves, with in-situ observations. Perspective and conclusions follow in section 4 .

\section{Proposed method}

Since the 1950s, with a turning point at the 1960 Ocean Wave Spectra conference (e.g. Longuet-Higgins et al., 1963), properties of ocean waves have been characterized routinely by spectra, estimated from time series, pictures or movies. This approach benefits from linear relationships between the wave elevation and measured variables (accelerations, pressure, slopes, etc.). Here, because the SAR transformation can be strongly nonlinear, we will first attempt to estimate a plausible surface velocity field that can explain observed patterns in SAR image, instead of estimating a wave spectrum. It is thus a deterministic approach in the sense that we resolve 
the phases of the waves and their detailed shape. We still use a spectral analysis but as a first guess and for final verification of the image properties. In this section we will neglect the blurring effect due to short wave components. That effect may be important close to the ice edge, as will be discussed in section 3 .

We have analyzed over 35000 wave mode images from two satellite missions Sentinel $1 \mathrm{~A}$ and Sentinel 1B that contain wave patterns in sea ice. A visual inspection reveals that in all these cases, the wave field is dominated by one or two narrow swell systems. We have thus developed a two-step algorithm which aims at estimating a likely field of orbital vertical velocities that can produce the observed image intensity. In this algorithm, we decompose the velocities into $v=v_{1}+v_{2}$, where $v_{1}$ is the velocity associated with swell number 1 propagating along a nearazimuth direction and creating bright lines in the SAR image, while $v_{2}$ is velocity of swell number 2 , propagating along a near-range direction and introducing wiggles in the lines when both swells are present. If these wiggles are absent, the first step of the algorithm is ignored. The success of this algorithm is measured by the differences in spectra normalized root mean square error between a simulated image and the recorded image.

A flow chart in Figure 2 represents the successive steps of the algorithm. The preprocessing step that produces multiple looks from a complex SAR image, containing radar amplitude and phase information, is now a standard processing technique (e.g. Engen and Johnsen, 1995). That step is required to remove the $180^{\circ}$ ambiguity on the wave propagation direction. Otherwise the algorithm can be applied to amplitude images, but without this ambiguity removal.

In this section we work exclusively with wave

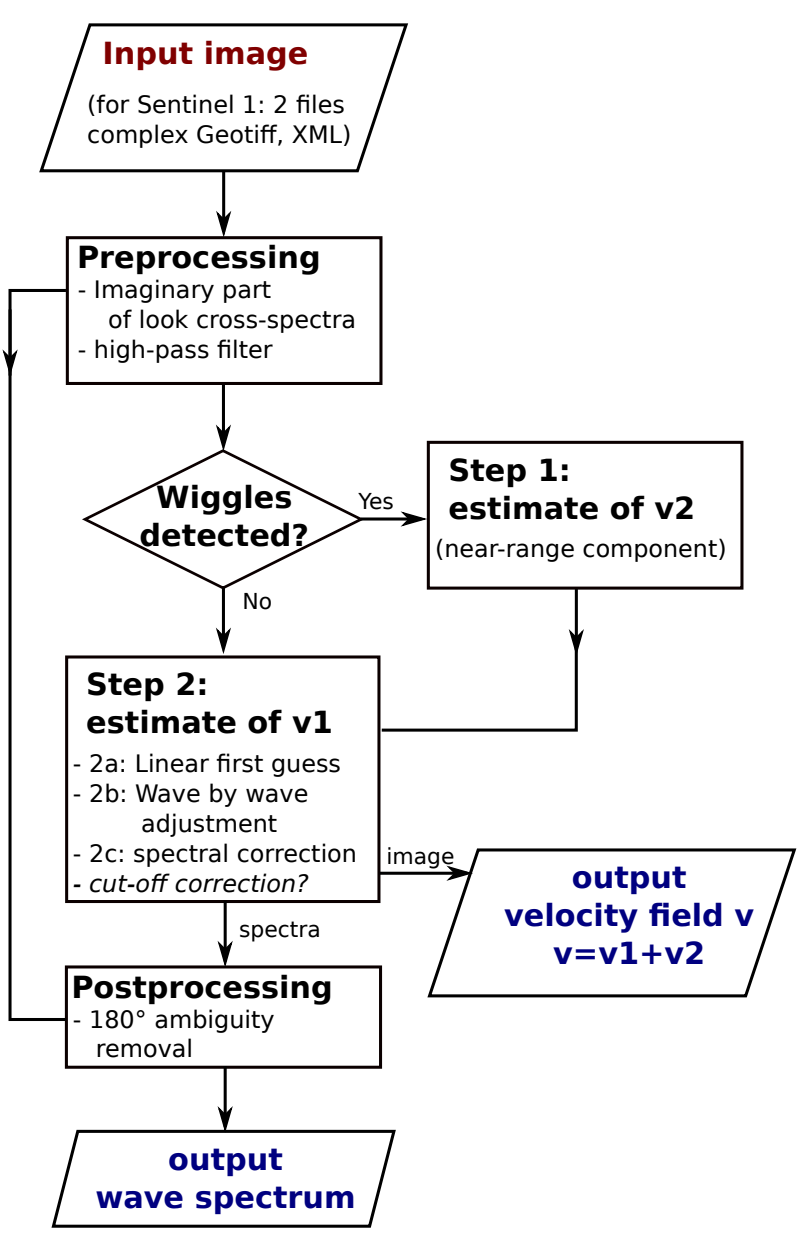

Figure 2: Different steps in the image processing that are detailed in the following subsection. The possible cutoff correction appears in italic because it has not been implemented yet and its feasibility is discussed in section 3. Without this cut-off correction the wave amplitudes of the $v_{1}$ component can be underestimated near the ice edge. 
mode data from Sentinel 1, which has a pixel resolution approximately $4 \mathrm{~m}$. We will, in particular, give details of the processing for the Sentinel $1 \mathrm{~A}$ wave mode image acquired on September 9, 2014, at 14:26:51 UTC, corresponding to Figure 1.e in Ardhuin et al. (2015). That image proved particularly difficult to process due to the presence of waves traveling near the range direction.

\subsection{Pre-processing}

The complex image is processed in three looks to estimate the real part and imaginary parts of cross-spectra between looks. This is performed following Chapron et al. (2001); Johnsen and Collard (2009), and only the imaginary part is stored to estimate the wave propagation direction without $180^{\circ}$ ambiguity. Otherwise all the processing is done on the amplitude image given by the modulus of the complex image. Starting from that amplitude we first apply a high-pass filter to remove large scale patterns in the image that can be caused by ice properties. This is performed by dividing the image intensity by its low-pass filtered value with a half power at $1 \mathrm{~km}$ wavelength.

This high-pass filtered image is then Fourier transformed to determine the dominant wavenumber $k_{1}$ and $k_{2}$ of the two possible swell systems. We first start with $k_{2}$, the dominant wavenumber of near-range swells, if any is present.

\subsection{Step 1: Inverting for $v_{2}$}

The first step is only applied if wiggles are present, namely when bright lines are not straight. Except if all the wiggle-related wave field propagates exactly in the range direction, the presence of wiggles should be associated with a peak in the image spectrum near the range direction. Our first step consists of finding the vertical velocity field $v_{2}$ that would produce the observed wiggles in a SAR image in the presence of straight features if they are present. The presence of that peak is detected using the following conditions: a maximum spectral density of the normalized image larger than $20 \mathrm{~m}^{-2}$, such that the peak wavenumber vector $\left(k_{p x}, k_{p y}\right)$ has $0.7\left|k_{p x}\right|>\left|k_{p y}\right|$ and a magnitude $k=\sqrt{k_{x}^{2}+k_{y}^{2}}$ larger than 0.01 , meaning that waves with periods longer than $20 \mathrm{~s}$ will be excluded. This filtering is similar to what is done for excluding non-wave patterns in wave mode data in open water (Johnsen and Collard, 2004), and can be relaxed if necessary. We first attempted an estimation of $v_{2}$ based on the known SAR transformation in the Fourier domain. This estimation failed because the transfer function goes to zero for waves propagating exactly in the range dimension, giving no image contrast for waves propagating in range. Still these waves have a clear effect on the image as they distort the other patterns. Our algorithm is thus based on measuring this distortion.

Every 5 pixels in range, at position $i$ and 5 pixels in azimuth at position $j$ we correlate a $160 \mathrm{~m}$ long segment in azimuth of the image, from $\left(i, j_{1}\right)$ to $\left(i, j_{2}\right)$ with similar segments shifted in azimuth. This shifted segment is taken at ranges $i-N_{i}<i^{\prime}<i+N_{i}$. Here we have taken $N_{i}=5$.

For each range $i^{\prime}$ we find a maximum correlation that corresponds to a shift $J\left(i^{\prime}\right)$. This gives the azimuthal relative displacement

$$
\delta_{0}(i, j)=\frac{1}{2 N_{i}} \sum_{i^{\prime}=i-N_{i}, i^{\prime} \neq i}^{i+N_{i}} \frac{J\left(i^{\prime}\right)}{i^{\prime}-i} .
$$

After a filter is applied in Fourier space to remove very large scale and very small scale shifts, these relative shifts are transformed to gradients 
(a)

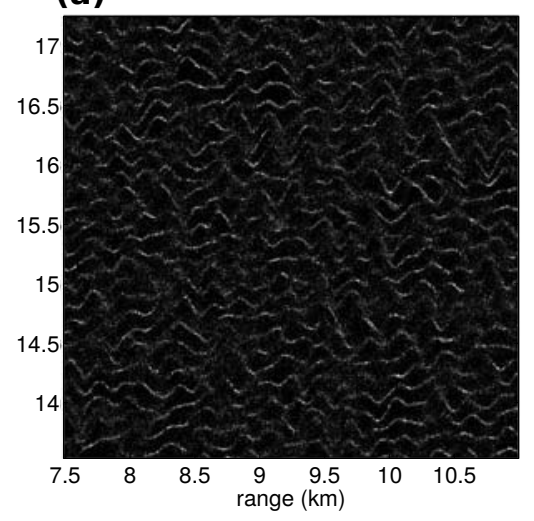

(b)

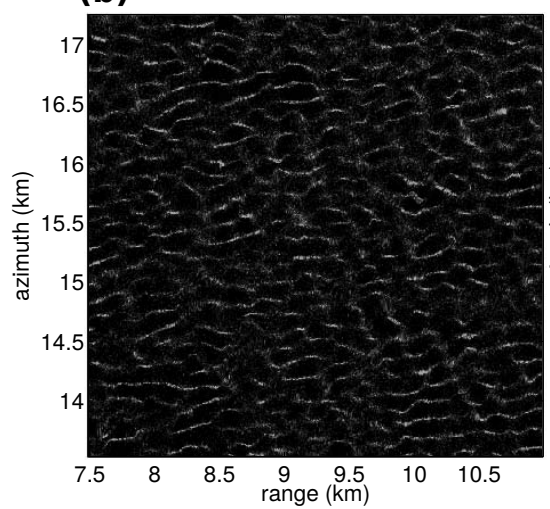

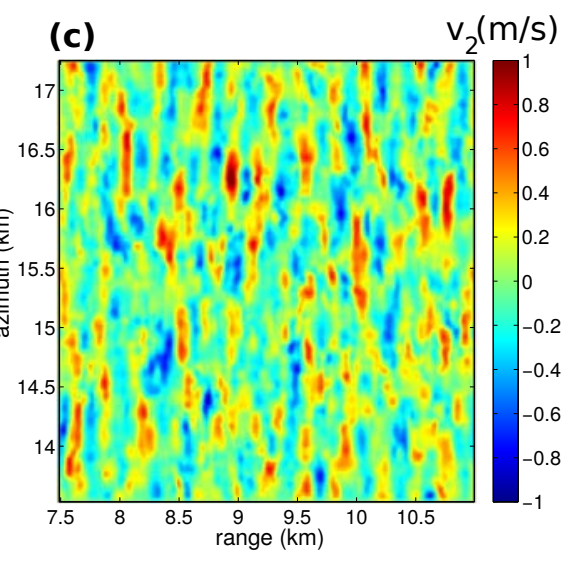

Figure 3: Illustration of the first step: the original image in (a) is transformed in (b) by an inverse SAR transformation given by eq. (3) using the velocity field $v=v_{2}$ shown in (c). The straightening algorithm works best for sharp and bright features, e.g. around $x=7.7 \mathrm{~km}, y=14.2 \mathrm{~km}$. The linear gray-scale for the image intensity goes from 0.5 to 8.

of $v_{2}$ as follows

$$
\frac{\partial v_{2}}{\partial x}=\frac{V}{Z} \delta_{0}(i, j)
$$

This relationship is also inverted in the Fourier domain to provide the $v_{2}$ field.

Once $v_{2}$ is determined, the SAR image $I_{S A R}$ is transformed into $I_{S A R}^{\prime}$ by displacing the pixels in the azimuth direction based on eq. (2) in which $v$ is replaced by $-v_{2}$. The result is that the original image, for example in Figure 3.a, is transformed into an image with much straighter lines, as shown in figure 3.b. The corresponding velocity field $v_{2}$ is shown in Figure 3.c.

This automatic processing likely underestimates $v_{2}$, as some wiggles remain. Further, the estimated waves tend to travel exactly in range, and the processing does not follow oblique crests very well. Some difficulty also comes from crossing lines: for example, around $x=10.3 \mathrm{~km}$ and $y=14.2 \mathrm{~km}$, wiggles of neighboring lines almost cross, because of the oblique orientation of the waves at that location, and the algorithm fails to follow the bright lines.

\subsection{Step 2: Inverting for $v_{1}$ \\ 2.3.1. Sub-step 2.a, first guess using Fourier transforms}

Starting from this straightened image (e.g. Figure 3.b), the second step attempts to find, for each range line $i=i_{0}$, a velocity $v_{1}\left(i_{0}, j\right)$ which produces the intensity distribution $I_{S A R}^{\prime}$. The distortion of the image given by eq. (1) results in a image pattern that is a non-linear transformation of the surface elevation, as shown in Figure 1.f, and Figure 2 of Alpers and Rufenach (1979).

Following these authors, we may start with the SAR transformation given by eq. (3) applied to a monochromatic oscillation in the azimuth, of complex amplitude $U$ and wavenumber $k_{y}$,

$$
v_{m}=\mathcal{R}\left\{U \exp \left(\mathrm{i} k_{y} y\right)\right\}
$$

where $\mathcal{R}$ stands for the real part. This gives a displacement

$$
\delta_{m}=\mathcal{R}\left\{Z U / V \exp \left(\mathrm{i} k_{y} y\right)\right\} .
$$


The important parameter for image patterns is the coefficient $c$ in Alpers and Rufenach (1979),

$$
C_{A R}=k_{y} U Z / V .
$$

Neglecting other effects, which means that we also assume a uniform backscatter, the SAR image intensity given by the Jacobian of the transformation $y \rightarrow y^{\prime}=y+\delta$, given by eq. (21) in Hasselmann and Hasselmann (1991) is

$$
J=\left|d y^{\prime} / d y\right|,
$$

for the monochromatic wave example that gives,

$$
I_{S A R}^{\prime}(y)=\frac{1}{\left|1+\mathcal{R}\left\{\mathrm{i} C_{A R} \exp \left(\mathrm{i} k_{y} y\right)\right\}\right|} .
$$

The amplitude of the velocity is $|U|=\sigma a$. For deep water waves propagating in the azimuth direction and neglecting ice effect on the wave dispersion, we have $\sigma=\sqrt{g k_{y}}$ where $g$ is the acceleration of gravity. These relations can be used to express $C_{A R}$ as a function of the wave height and period.

There is thus a linear transformation from the velocity to the SAR intensity for $C_{A R}<<1$. It should be noted that $C_{A R}=1$ corresponds to the case in which caustics appear. In our Figure 1.f, with a wave period of $10 \mathrm{~s}$ traveling in the azimuth direction, $C_{A R}=1$ corresponds to an elevation amplitude $a=0.42 \mathrm{~m}$. Random waves of the same energy would have a significant wave height $\left.H_{s}=4 \sqrt{(} a^{2} / 2\right)=1.2 \mathrm{~m}$.

For narrow wave spectra, we can define $C_{A R}$ using a root mean square velocity in eq. (8). This parameter clearly defines several regimes, as illustrated by the variation of the image normalized variance (solid lines) and minimum intensity (dashed lines) shown in Figure 4. For $C_{A R}>1$, each bright line becomes a doublet. The two lines of the doublet progressively drift apart as the amplitude increases, pulling the minimum intensity to lower and lower values, up to the point where lines from different doublets meet, at $C_{A R} \simeq 4.6$. Beyond this value there is no region of very low intensity, producing an increase in the minimum intensity and a decrease in the normalized variance (Figure 4.b). At that level of non-linearity a unique mapping that produces a given SAR image is unlikely. Other
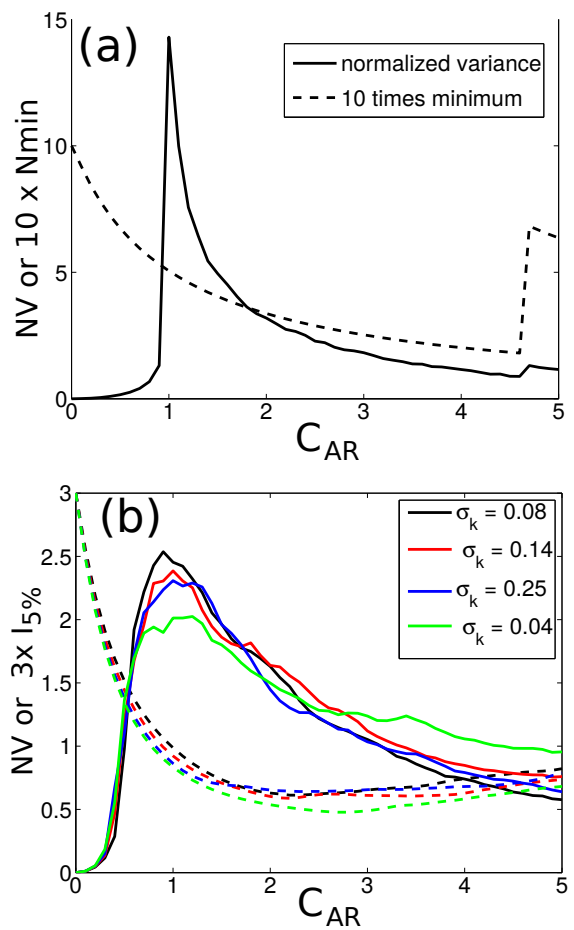

Figure 4: (a) Variation of the normalized image variance and normalized minimum intensity in the case of a monochromatic wave of amplitude $a$ traveling along the azimuth direction, as a function of the SAR mapping nonlinearity parameter. (b) Similar variation but for a Gaussian wavenumber spectrum $E(k) \propto \exp (-((k-$ $\left.\left.k 0) / \sigma_{k}\right)^{2} / 2\right)$, with $C_{A R}$ defined from the r.m.s. wave amplitude. $I_{5 \%}$ is the mean intensity of the $5 \%$ less intense pixels, shown with dashed lines.

parameters of the wave spectrum, such as the 
spectral width $\sigma_{k}$, have a relatively minor influence on the distribution of the image intensity, as shown in Figure 4.b.

Several authors have used a quasi-linear inversion scheme, for example Hasselmann and Hasselmann (1991), Krogstad (1992) and Forget et al. (1995), combining a linear transfer function for the constructive velocity bunching, and an attenuation for the blurring effect caused by the velocity bunching associated with short waves. For $C_{A R}<0.5$, we are nearly in the linear regime, and we may invert eq. (10) directly. First ignoring blurring by short waves this gives,

$$
v_{1, \text { lin }}=C_{l} \quad \operatorname{IFT}\left(\widehat{I}_{S A R}^{\prime}\left(\mathrm{i} k_{y}\right) \frac{V}{Z k_{y}} U\left(k_{y}\right)\right),
$$

where we use $C_{l}=0.5$. IFT stands for the inverse Fourier transform, $\widehat{I}^{\prime}$ is the Fourier transform of $I^{\prime}$ along the azimuth, and $W$ is a bandpass filter around the swell wavenumber. The correction for $C_{l}$ and filters is performed later in step 2.c.

\subsection{Inverting for $v_{1}$ : Step 2.b further wave-by- wave adjustment}

In general, even for $C_{A R}>0.5$ we will still use eq. (11) but this is now only a first step to a non-linear inversion. When $C_{A R}$ approaches 1, using $C_{l}=1$ tends to overestimate the doubling of lines, which is why we start with $C_{l}=0.5$. The velocity field $v_{1, \text { lin }}$ gives a quasi-linear modeled SAR image $I_{l}^{\prime}$. One example is shown in blue in Figure 5, corresponding to the range $x=7.488 \mathrm{~km}$ in Figure 3, with the modeled intensity in the top panel, corresponding to $v_{1, \text { lin }}$ shown in the bottom panel.

In Figure 5.a, the most obvious difference between the intensity modeled from $w_{1, \text { lin }}$ in blue and the real image in black, is the lack of very low values and the small width of the peaks. This is typical of images with values of $C_{A R}$ between 0.3 and 0.8. Because the pixels are only displaced, the mean image intensity is conserved. A simple way to produce lower minima is thus to shift further the pixels from the low value regions to the peaks. This change of the intensity distribution corresponds to the regime $C_{A R}<2$, as shown in Figure 4.

In order to adjust the low intensities, we perform a wave by wave analysis in azimuth, adjusting each wave shape to produce the desired intensity. We define segments as being between two consecutive zeros of the second derivative of $v_{1, \text { lin }}$. Each segment generally contains a short region of values of $I_{S A R}^{\prime}$ above 2 and often up to 10 . This region is the bright line centered on $y_{m}$. The intensity around $y_{m}$ is usually underestimated with a value $I_{l}^{\prime}$. In order to enhance $I_{l}^{\prime}$, we adjust the mean intensity in the two regions of the segment on either side of the bright line, where the normalized image intensity is less than 1.5. This is done by adding a velocity increment shaped like a Hanning window over each region. Each increment is proportional to the difference between the mean of $1 / I_{S A R}^{\prime}$ and the mean of $1 / I_{l}^{\prime}$ of that region. For $y<y_{m}$ a positive increment of $w$ shifts more intensity to the bright line, and for $y>y_{m}$ a negative increment of $w$ also reinforces the bright line. The result of this correction is shown with red lines in Figure 5.

After these adjustments, the exact position of the bright line may be slightly shifted by $\delta_{m}$, and this is corrected by adding one more velocity increment that is zero on the boundaries of the segment and takes the value $-\delta_{m} V / Z$ near $y_{m}$. 

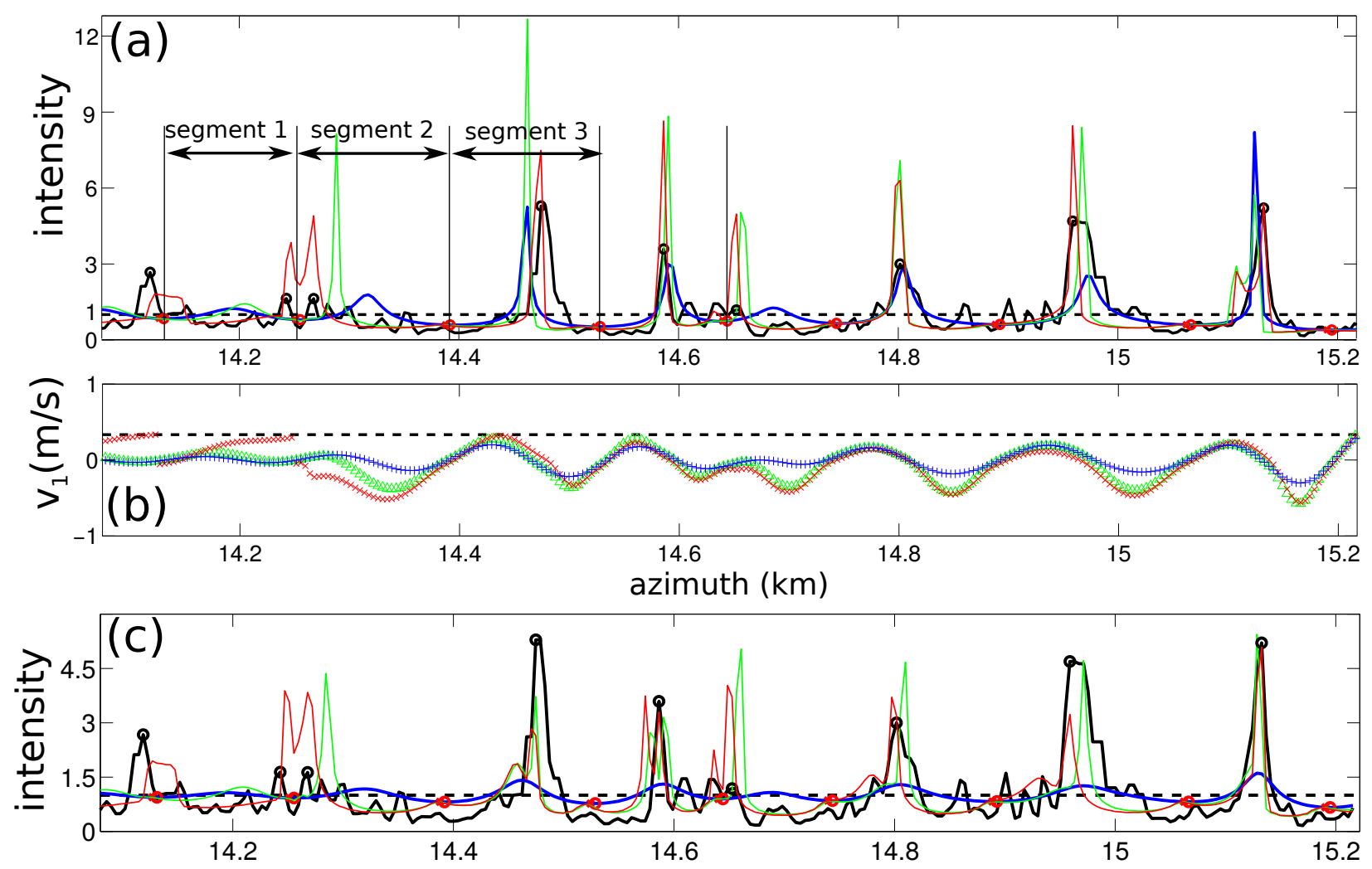

Figure 5: (a) Example of image intensity as a function of the azimuth for a fixed range (here at $\mathrm{i}=1$, corresponding to $x=7.488 \mathrm{~km}$ ), starting from $C_{l}=0.7$, and (b) corresponding adjustments of the vertical velocity $v_{1}$. Starting from a linearized inverse SAR transform that gives $v_{1, \text { lin }}$ in blue, the next adjustments include a correction for the low intensity values (in green), a shift of the peaks (in red). (c) is the same as (a) but using a lower linear first guess, with $C_{l}=0.3$. The black circles mark the peaks of the image intensity used to define wave segments for the nonlinear adjustment of the velocity $v_{1}$.

\subsection{Inverting for $v_{1}$ : Step 2.c final adjustment based on image spectra}

The result of steps 2.a and 2.b is a velocity field that is filtered around the peak of the image spectrum. A simulated SAR image is produced and both this image, and the image obtained after step 1 , are filtered in the same way to produce a correction factor $R$. We define $R$ as the ratio of the square root of the filtered image variance in the SAR image and the variance in the filtered simulated image. This correction is applied to the first guess $v_{1, \text { lin }}$ and steps 2.b and 2.c are repeated a second time. In principle, the simulated and measured image variance around the peak wavenumber $k_{1}$ should be similar. This works particularly well for $C_{A R}<0.6$.

After these steps, a simulated image such as shown in Figure 6.b, is expected to resemble the original SAR image. This simulated image is based on a velocity field $v=v_{1}+v_{2}$, and that velocity field is our estimate of the wave-induced orbital velocity, from which we may estimate a 

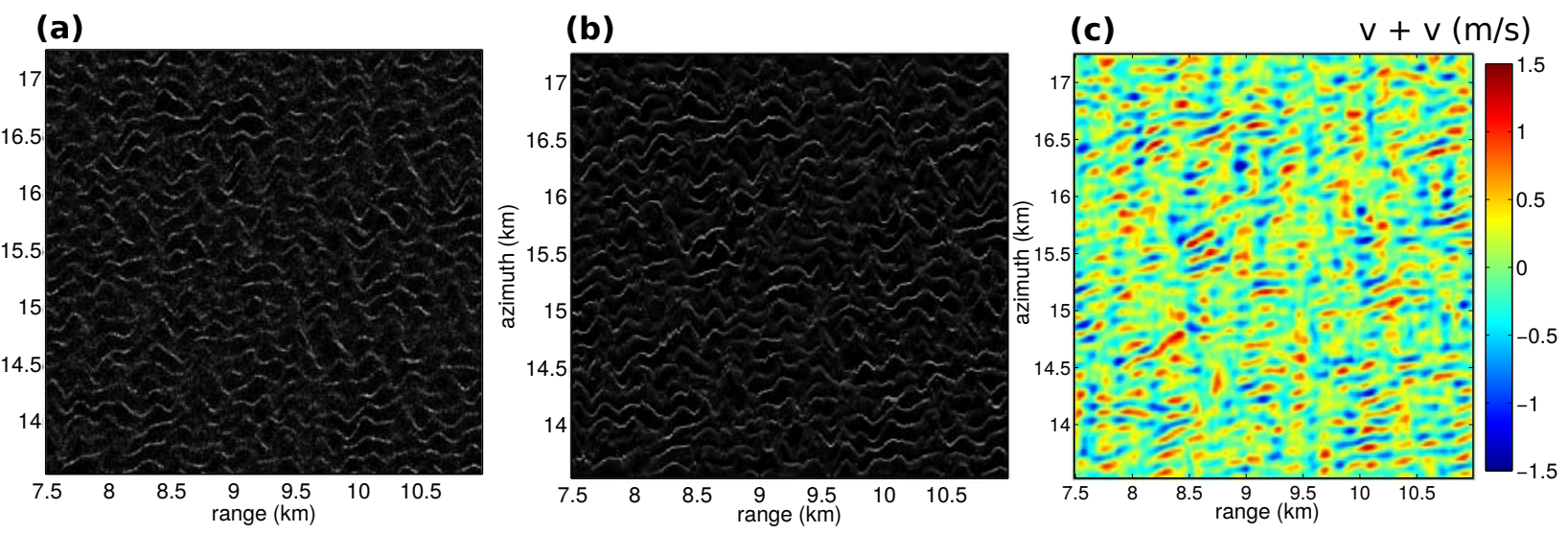

Figure 6: Illustration of the combination of step 1 and step 2: the vertical velocity field $v_{1}+v_{2}$ shown in (c) produces a simulated SAR image (b) that looks like the recorded image (a).

wave spectrum (Fig. 7). So far we only used the SAR image amplitude, and the propagation direction of the waves has a $180^{\circ}$ ambiguity.

\subsection{Spectral post-processing: ambiguity removal}

Following exactly the procedure of the wave mode processing to obtain level 2 spectra, we estimated the imaginary part of the image cross spectra from the full 20 by $20 \mathrm{~km}$ image, using Fourier transforms over 4 by $4 \mathrm{~km}$ tiles. We have taken the average for all tiles and the average of the cross-spectra between look pairs 1 and 2, and 2 and 3 . Each spectral component pair $(k, \theta)$ and $(k, \theta+\pi)$ is dominated by waves coming from the direction with a positve imaginary part of the cross spectrum. Assuming that there is energy in only one of these two components we can then produce a non-ambiguous directional spectrum. The directions obtained here are consistent with the numerical model results shown in Ardhuin et al. (2015).

Figure 8 shows a very clear direction for the two main swell peaks. There is a sign reversal for the longest components, with wavelength of
$600 \mathrm{~m}$ which according to the SAR image phase comes from direction $30^{\circ}$ relative to range instead of $210^{\circ}$ according to our numerical model. Such a sign reversal for very long components has also been found in open ocean Sentinel 1A data in 2014 and has been attributed to a processing artefact in forming the level 1 SAR image. This artefact is apparently corrected in more recent data from 2016. To mitigate the effects of such artefacts and possible noise, Johnsen and Collard (2009) use a wave spectrum partitionning scheme on the image spectrum, and only keep those partitions that have an average positive imaginary cross-spectrum.

\subsection{Verification}

An error measure based on the difference between the modeled and measured image is not very practical because of all the small scale details in the image which can be misplaced. Another approach is to measure the error on images filtered around the scales of the velocity field. However, this filtering removes the non-linear signature of the SAR transformation. We have 

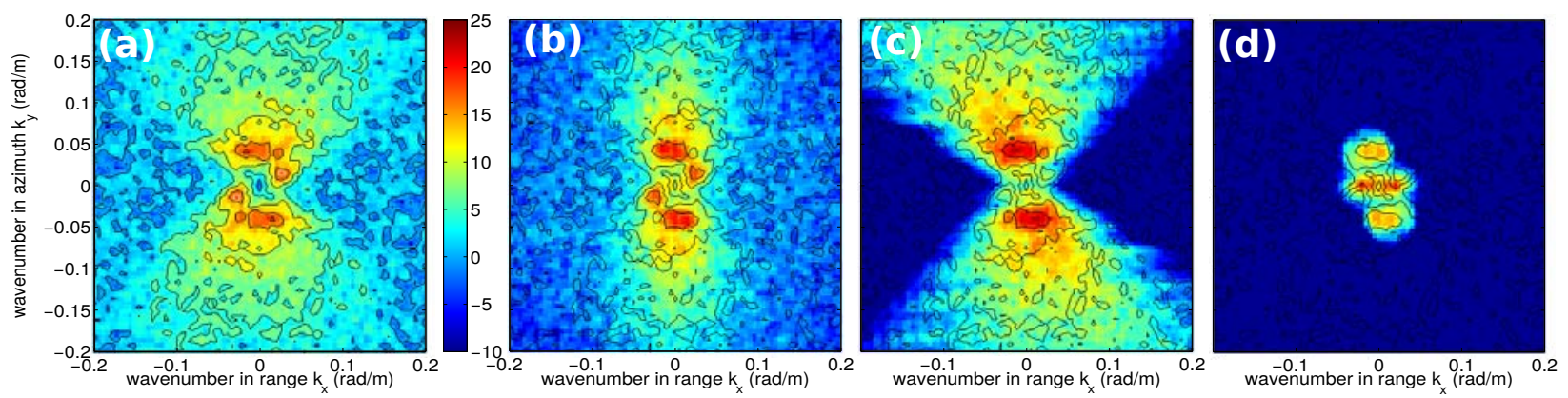

Figure 7: (a) Power spectrum of image amplitude in figure 3.a, (b) spectrum of straightened image in Figure 3.b, (c) spectrum of simulated SAR image in Figure 6.b, (d) spectrum of velocity in Figure 6.c. All spectral densities are in $\mathrm{dB}$ relative to $\mathrm{m}^{-2}$ for the images or $\mathrm{s}^{-2}$ for the velocities. Colors show the power spectra of each quantity, and the overlaid contours correspond to contours of the image spectrum (a) in order to better visualize the differences in spectral shape.

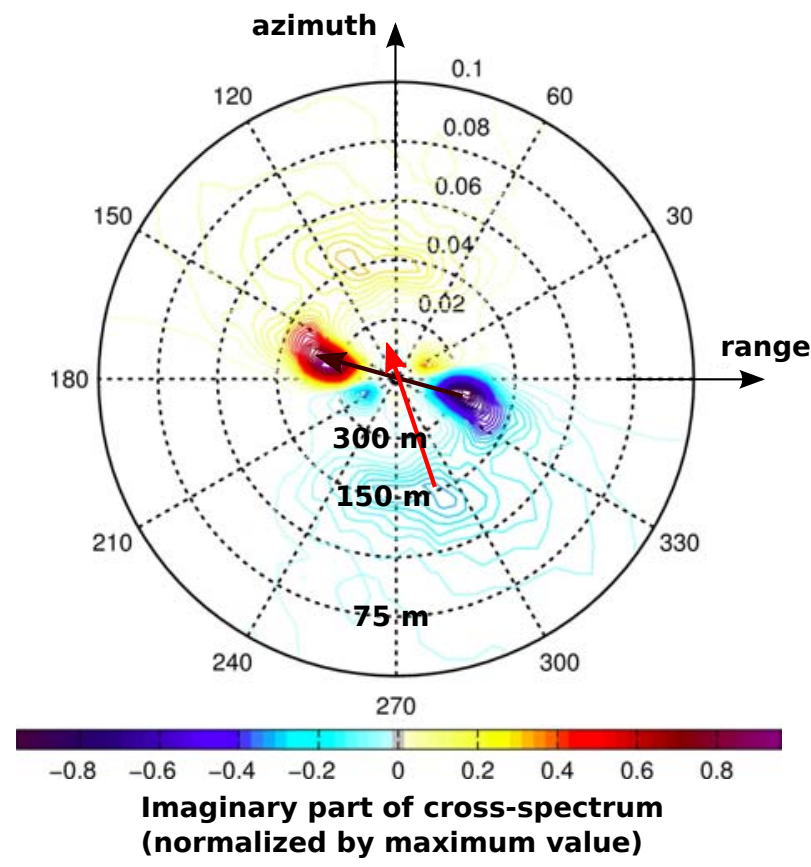

Figure 8: Imaginary part of the cross spectrum between pairs of image looks separated by $0.3 \mathrm{~s}$, that reveals the overal displacement of the wave patterns between the looks. The inferred direction of propagation of the two main swell peaks are represented by the two arrows. Values on the rings are wavenumber $k$ in $\mathrm{rad} / \mathrm{m}$ and wavelengths in meters. thus essentially worked with the differences in spectra between the SAR image spectrum $E_{S A R}$ and the modeled image spectrum $E_{\text {mod }}$ to define the error,

$$
e=\frac{\sum\left|E_{\text {mod }}\left(k_{x}, k_{y}\right)-E_{S A R}\left(k_{x}, k_{y}\right)\right|}{\sum\left|E_{S A R}\left(k_{x}, k_{y}\right)\right|},
$$

where the sum is restricted to the spectral components with $E_{\text {mod }}>0.01 E_{\text {mod,max }}$.

In the difficult case used as an example in this section, with near-range components, the simulated spectrum clearly fails to produce a strong enough variance at the same position as the nearrange peaks, giving and error $e=0.84$. We expect that better methods or a better tuning of our step 1 may be able to better represent that peak.

\subsection{Another example: quasi-linear case}

We show here another example from S1A wave mode images. One example of a nearly linear image, shown in figure 9 , was acquired on the same orbit a little further in the ice, about $300 \mathrm{~km}$ from the ice edge. This case is very nearly linear and the image variance around the spectral 
peak $k_{2}$ in the simulated image exactly matches the observed value. For that image the spectral error is $e=0.58$. This linear inversion of the image spectrum gives a significant wave height of $1.3 \mathrm{~m}$.

This value corresponds to a small piece, $3.5 \mathrm{~km}$ on the side, of the entire wave mode image. The piece by piece processing of the full image shown in Figure 10.a gives the map of wave heights in Figure 10.b. The image exhibits elongated dark features that are probably water channels, known as leads, between large ice floes with typical sizes of the order of 5 to $10 \mathrm{~km}$. These features suggest that either the ice has not broken into smaller floes, or that it has just broken and has not yet deformed.

The high pass filter removes most of these dark features and the spectrum is only weakly contaminated giving a variability in wave height that is probably dominated by the sampling error for a random sea state. The map of wave heights exhibits apparently random fluctuations that must be related, at least in part, to the sampling of the random wave field. From Figure 9 we can estimate that each sub-image contains about 20 wave crests. Using a Rayleigh distribution for the wave height, this gives an expected $11 \%$ standard deviation of the estimated wave height, which is consistent with most local variations. However, the jump from 0.9 to $1.3 \mathrm{~m}$ at $y=4 \mathrm{~km}$ around $x=10 \mathrm{~km}$ is also probably due to the lead visible as a darker region in Figure 10.a. Besides these local fluctuations, there is a clear gradient in azimuth from an $H_{s}$ value around $1.2 \mathrm{~m}$ at small azimuths, decreasing to values around 0.8 at the largest azimuths, further away from the ice edge. This $30 \%$ reduction of $H_{s}$ over $15 \mathrm{~km}$ is a factor 5 stronger than the average attenuation from the ice edge to this location. It may be caused by the stronger in- teraction of ice and waves when the ice is made of floes larger than the wavelength (e.g. Collins et al., 2015; Ardhuin et al., 2016).

\section{Blurring by small scale motions}

Here we apply the algorithm described above to pieces of an Interferometric Wide swath image (IW mode) acquired by Sentinel-1A on November 1, 2015 at 17:23 UTC, in the Beaufort Sea, north of Barrow, Alaska. This specific acquisition was requested from the European Commission and ESA, instead of the usual Extended Wide swath usually used in this region, in order to take advantage of in situ deployments for the validation of new methods to estimate waves and ice parameters from SAR imagery. The full set of images can be viewed at http://bit.ly/22JFruo. This experiment was part of the Sea State and Boundary layer physics program of the Office of Naval Research (Wadhams and Thomson, 2015). The in situ measurements include directional wave spectra derived from buoys, directional spectra from a marine radar, and a laser rangefinder on $\mathrm{R} / \mathrm{V}$ Sikuliaq; more details can be found at http://apl.uw.edu/arcticseastate .

The context of the November 1 image and available in situ data is given in Figure 11. All the in situ measurements on that day are within $6 \mathrm{~km}$ of the ice edge. The Sentinel 1A image was acquired after a low pressure system passed over the ship, traveling from south-west to northeast. On October 31, the wind was mostly from the South-East, turning from 160 to $105^{\circ}$ (nautical convention) throughout the day. At the time of the SAR image, the wind was blowing from the South-East (120 degree nautical) along the ice edge. This condition gives a directional wave spectrum in the open water, shown 

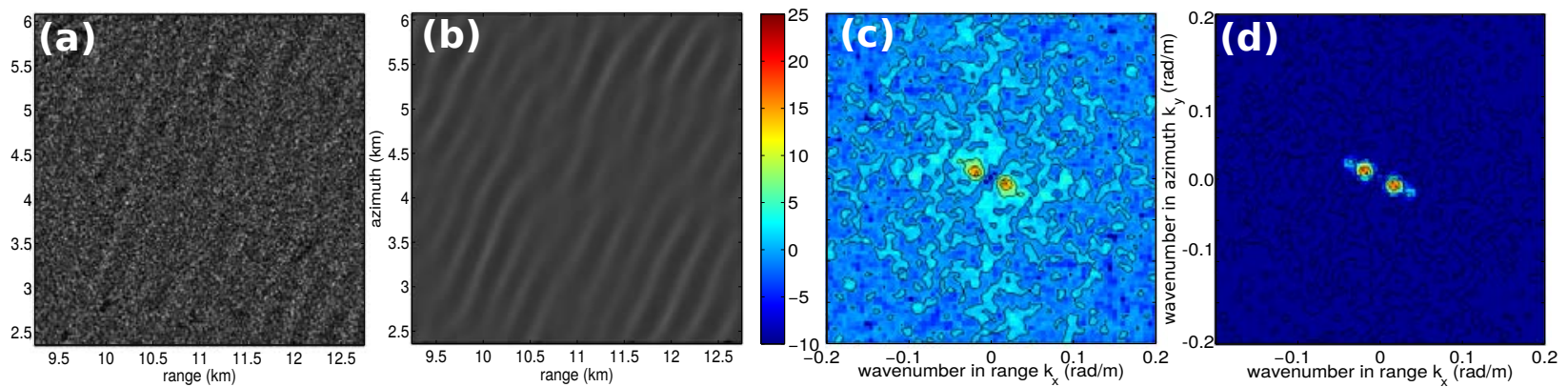

Figure 9: (a) Piece of a S1A wave mode image acquired on September 9, 2014, at 14:27:20 UTC centered at 62.96 S, $148.60 \mathrm{E}$ (b) simulated image, (c) image spectrum, (d) spectrum of simulated image. In this case the image is nearly linear with $C_{A R}=k_{y} Z\left|U_{\mathrm{rms}}\right| / V=0.35$ and step 2.b is not necessary. The linear gray-scale for the image intensity goes from 0 to 4 . Colors show the power spectra of each quantity, and the overlaid contours correspond to contours of the image spectrum (a) in order to better visualize the differences in spectral shape.

in Figure 11.d, that is typical of coastal environments with winds blowing obliquely alongshore. Namely, the highest frequencies propagate downwind, while the mean wave direction gradually moves towards the ice-edge-normal direction at lower frequencies, due to a stronger importance of fetch and propagation compared to local forcing (e.g. Ardhuin et al., 2007), and the propagation of swell from the previous day. The ice around R/V Sikuliaq was newly formed first-year ice with an estimated thickness of 10$15 \mathrm{~cm}$ determined from visual observations and samples collected with a basket.

Figure 11.b,c shows that waves are hardly visible in the open water at the location of buoy S15, and become progressively more visible at locations further into the ice, for example buoys $\mathrm{S} 13$, W3, or the location of R/V Sikuliaq. In order to apply our algorithm we chose a region of relatively uniform modulation, shown in Figure 12 , very close to $\mathrm{R} / \mathrm{V}$ Sikuliaq but without the ship in the image, to avoid a contamination of the wave parameters by the radar signature of the ship. At the time of image acquisition, the ship is located at range $=117.5 \mathrm{~km}$, azimuth $=$

\section{$70.8 \mathrm{~km}, 1 \mathrm{~km}$ to the left of 12.a.}

The laser rangefinder measured time series of surface elevation as it was steaming through the ice. This surface elevation is the water surface or ice elevation. Because the ice is thin, ice freeboard effects are expected to be less than $3 \mathrm{~cm}$, which we will neglect to directly compare this elevation to a wave-induced water elevation. Using an a priori estimate of wave direction from the buoys, the surface elevation spectrum in relative radian frequency $\sigma_{r}=\sigma+\mathbf{k} \cdot \boldsymbol{U}_{r}$ was converted to absolute frequency $f=\sigma /(2 \pi)$, where $\boldsymbol{U}_{r}$ is the ship velocity vector and $\mathbf{k}$ is the wavenumber vector. The SAR-derived wavenumber spectrum was also converted to frequency assuming linear wave theory in deep water without ice effects, namely $\sigma^{2}=g k$.

These different estimates of the elevation frequency spectra $E(f)$ are compared in Figure 13. Spectral levels at frequencies $f>0.2 \mathrm{~Hz}$ are significantly lower at buoy S13 than at S15, potentially due to the damping effect of the ice on the waves. The SAR-derived spectra decrease rapidly towards high frequency. A spectrum computed right at the ice edge around buoy 

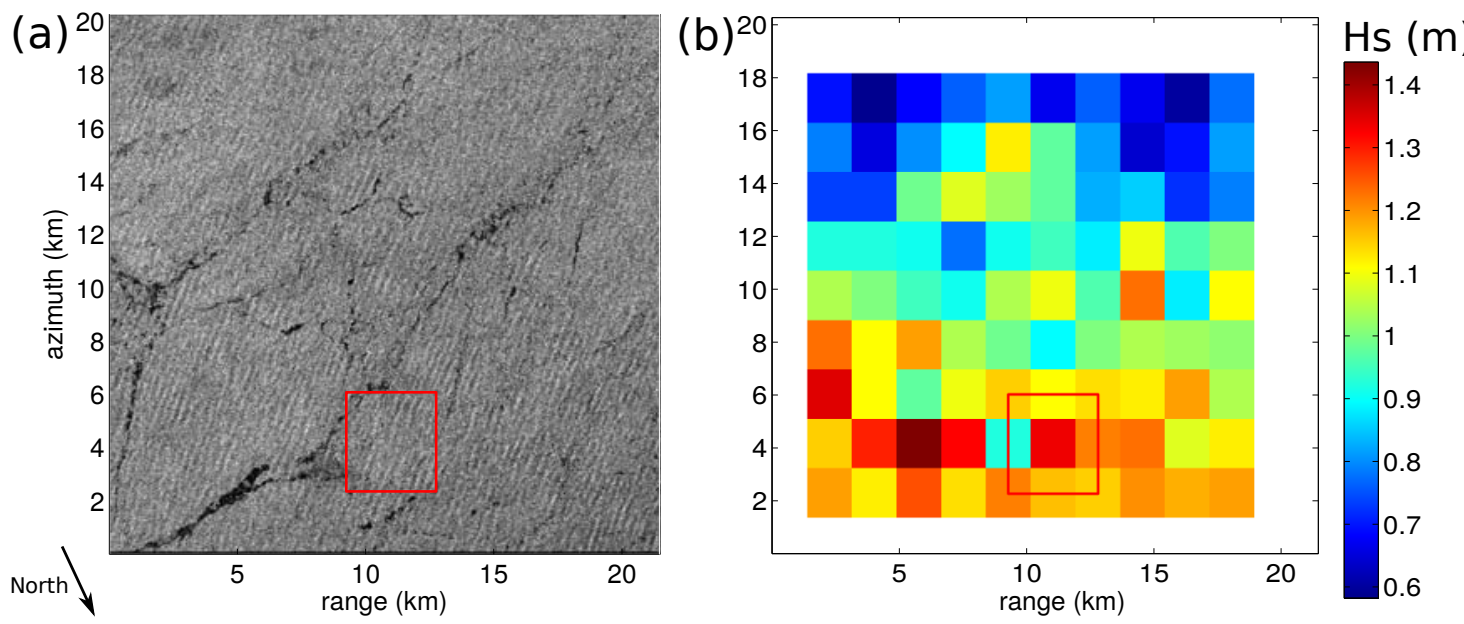

Figure 10: (a) Entire S1A wave mode image acquired on September 9, 2014, at 14:27:20 UTC, the image processing described here is performed on square sub-image, including the solid red square corresponding to the sub-image shown in Figure 9. (b) Map of significant wave heights determined from each sub-image.

S13 gives a significant wave height of $0.35 \mathrm{~m}$, compared to the $1.04 \mathrm{~m}$ measured by the buoy. This is a much lower energy than the one computed close to the ship, where the measured wave height of $0.76 \mathrm{~m}$ is larger than the SAR-derived wave height of $0.5 \mathrm{~m}$. The SAR-derived spectra also contain some spurious energy at low frequencies, below $0.1 \mathrm{~Hz}$, which may well be the contribution of ice features to the image spectrum. It is particularly difficult to define an image filter that catches the main spectral peak but removes larger scale non-wave features.

Clearly the estimated wave energy is much lower than measured by buoys and the laser rangefinder. This is likely due to the strong reduction in the image spectral variance due to unresolved short wave components, leading to the well known cut-off effect. A theoretical estimate of that effect is given by the quasi-linear approximation of Hasselmann and Hasselmann (1991), also re-derived by Krogstad (1992). From a second order approximation of the SAR-induced pixel displacement $\delta$, the image spectrum is given by eq. (56) in Hasselmann and Hasselmann (1991). Re-written with our notations, this gives a reduction of the quasilinear spectrum $E_{q l}$ compared to the linear spectrum $E_{l}$ by a factor that increases exponentially with azimuthal wavenumber, $k_{y}$, and defines the cut-off wavelength $\lambda_{c}$,

$$
\begin{aligned}
E_{q l}(\mathbf{k}) & \simeq \exp \left(-k_{y}^{2}<v^{2}>Z^{2} / V^{2}\right) E_{l}(\mathbf{k}) \\
& =\exp \left(-k_{y}^{2} \lambda_{c}^{2} /(2 \pi)^{2}\right) E_{l}(\mathbf{k}) .
\end{aligned}
$$

In this equation $\left\langle v^{2}\right\rangle$ is the vertical orbital velocity variance, with some correction for wave direction as discussed in section 1 .

This theoretical prediction was thoroughly tested by Kerbaol et al. (1998) with more details given in Kerbaol (1997). In particular he found that the velocity variance that should go into eq. (13) is not the full variance, but only that of waves shorter than a given threshold, which he found to range between 0.29 times the 

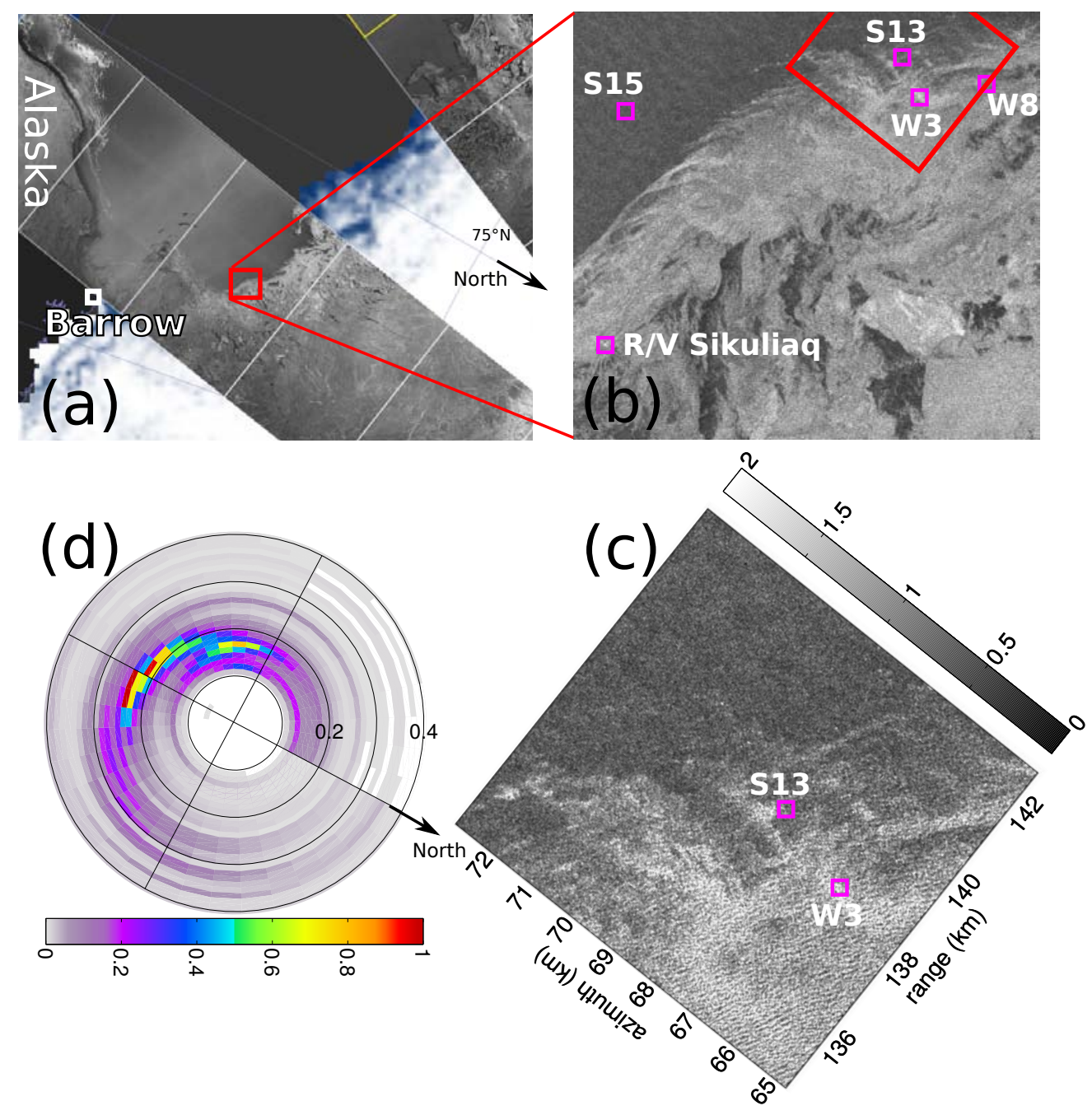

Figure 11: Context of the S1A IW image acquired on November 1st, 2015, at 17:23 UTC, and available in situ data. The full image at full resolution can be viewed at http://bit.ly/22JFruo . (a) SAR-derived roughness (gray scale) showing open water between the Alaska shoreline, to the West of Barrow, and the measurement locations in the red box. (b) Location of the R/V Sikuliaq and some of the measurement buoys. Note that buoy S15 is in open water. (c) piece of the SAR image around the buoys S13 to W3. (d) Directional spectrum estimated from S15 data using the Maximum Entropy Method (Lygre and Krogstad, 1986). 

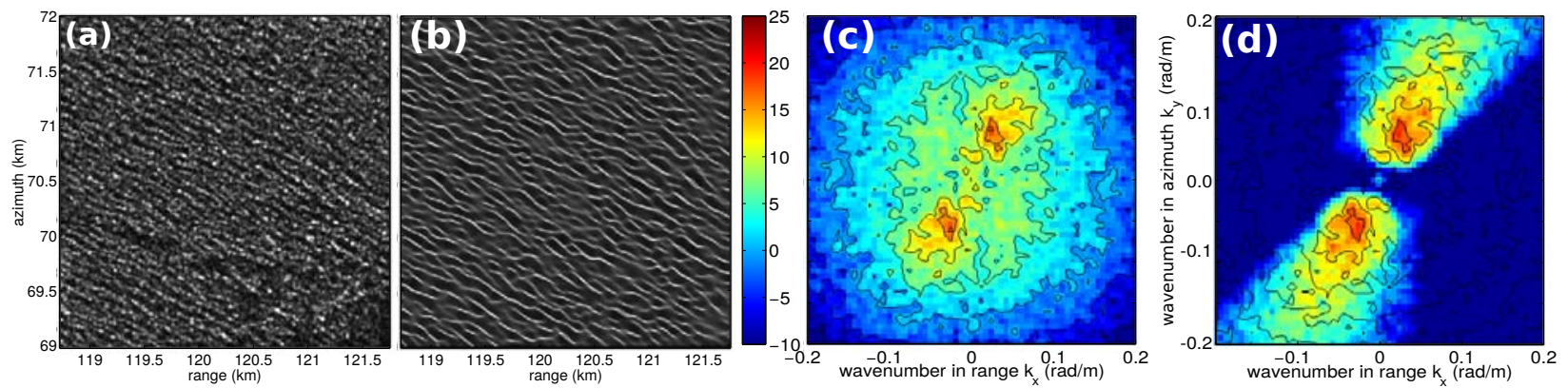

Figure 12: (a) Piece of a S1A IW image acquired on November 1st, 2015, at 17:23 UTC next to the position of $\mathrm{R} / \mathrm{V}$ Sikuliaq, (b) simulated image, (c) image spectrum, (d) spectrum of simulated image. In this case the image is strongly non-linear with $C_{A R}=0.95$ and step 2.b is necessary. The linear gray scale for the image intensity goes from 0 to 4 .

peak wavelength $\lambda_{p}$ for short waves propagating in the range direction, and 0.17 times $\lambda_{p}$ for short waves propagating in the azimuth direction. These coefficients were determined for waves in the open ocean and wave mode data from ERS-1. As the shape of the short wave spectrum is strongly affected by the ice cover the coefficient may be different and we will only use them here for illustration. With a peak frequency at $f_{p}=0.12 \mathrm{~Hz}$, the velocity variance of waves shorter than $0.29 \lambda_{p}$ is $0.025 \mathrm{~m}^{2} \mathrm{~s}^{-2}$ at buoy $\mathrm{S} 13$ and $0.018 \mathrm{~m}^{2} \mathrm{~s}^{-2}$ at buoy W3 compared to $0.13 \mathrm{~m}^{2} \mathrm{~s}^{-2}$ at $\mathrm{S} 15$. Using this restricted velocity variance $\left\langle v^{2}\right\rangle$ in eq. 13 gives a cutoff wavelength $\lambda_{c}=114 \mathrm{~m}$ at S13, $\lambda_{c}=87 \mathrm{~m}$ at $\mathrm{W} 3$, and $\lambda_{c}=44 \mathrm{~m}$ at $\mathrm{R} / \mathrm{V}$ Sikuliaq.

As shown in Figure 13, this theoretical estimate explains the apparent reduction in the image spectrum at the location of buoy S13, and thus the error in wave estimation if this blurring effect of the short waves is not taken into account. Without any correction for the blurring, the estimate wave height increases from $0.14 \mathrm{~m}$ in the open water to $0.35 \mathrm{~m}$ near buoy S13 and $0.52 \mathrm{~m}$ near the ship, contrary to the measurements from the in situ sensors which show a decay from $H_{s}=1.2 \mathrm{~m}$ at $\mathrm{S} 15$ and W3, to $H_{s}=0.76 \mathrm{~m}$ at $\mathrm{R} / \mathrm{V}$ Sikuliaq.

Far inside the ice, the short waves should be attenuated enough to neglect the blurring effect. We may use an expected spatial attenuation of short waves energy $\alpha=-d E / d x / E$, typically larger than $10^{-4} \mathrm{~m}$ for periods shorter than $10 \mathrm{~s}$, meaning that the wave energy is typically reduced to less than $2 \%$ of the open water at distances in the ice larger than $40 \mathrm{~km}$. At these distances, starting from an open water wave spectrum given by Pierson and Moskowitz (1964), the cut-off wavelength is $41 \mathrm{~m}$, giving a $5 \%$ reduction in wave height due to blurring for waves of $10 \mathrm{~s}$ period, an effect that can be neglected for most applications. The blurring effect is weaker for longer wave periods.

\section{Summary and perspectives}

We have demonstrated here the implementation of an algorithm to determine wave-induced orbital velocity maps from SAR images over icecovered regions. SAR images in the ice usually show one or two swell trains. Wave-induced features in the SAR images take the form of bright 

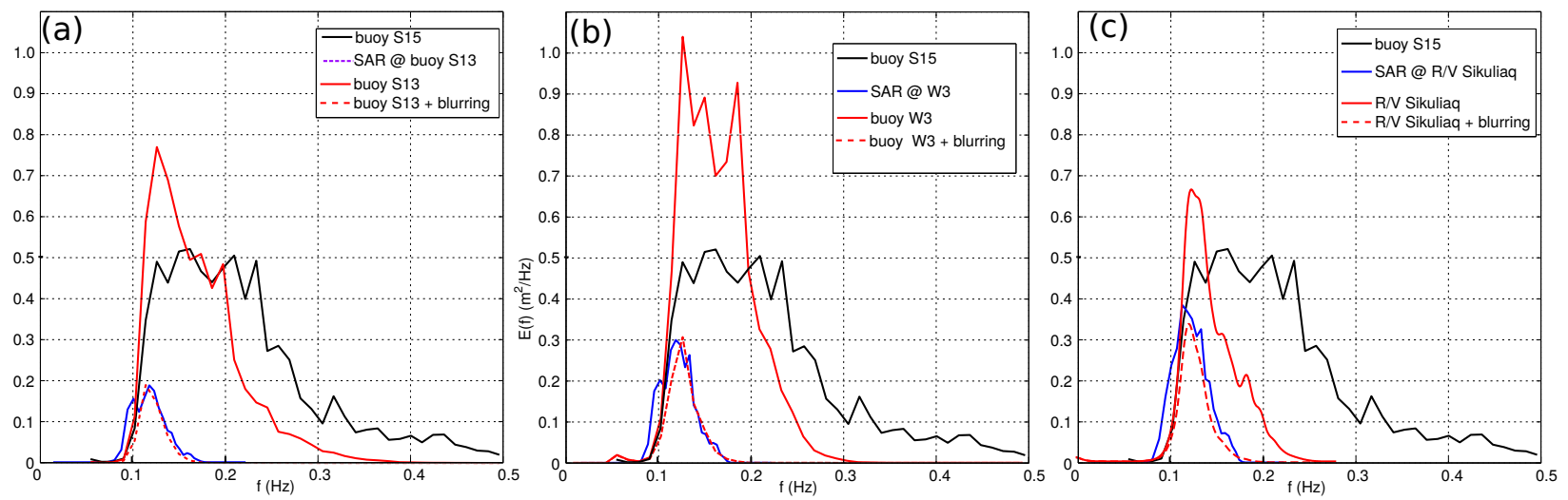

Figure 13: Comparison of spectra derived from in situ data and the SAR image around (a) buoy S13, (b) buoy W3, (c) near the ship position. In each panel the spectrum at the offshore buoy S15 is indicated for reference. The 'blurring' effect is the reduction of wave spectrum according to eq. (13).

lines which can be wiggled if one swell travels near the range direction. Our two step algorithm is expected to work best when the image nonlinearity parameter $C_{A R}=k_{y} Z\left|U_{\mathrm{rms}}\right| / V$ is small, typically less than 0.5 , where $k_{y}$ is the azimuthal wavenumber of the image pattern, $Z$ is the platform altitude above ground $\left|U_{\text {rms }}\right|$ is the root mean square orbital velocity of the wave motion, and $V$ is the platform speed with respect to the Earth.

This attempt at a fully non-linear inversion of the SAR mapping shows that the first step is a robust way to determine wave parameters in the presence of wiggles, and the second step can be used to derive wave parameters of a single swell, or the parameters of a second swell when wiggles are present.

Comparison with in situ data has shown that, close to the ice edge, short unresolved waves can cause a significant reduction in the estimated wave spectrum, and this reduction is well predicted by the theoretical cut-off of the quasilinear theory of Hasselmann and Hasselmann (1991), provided that the velocity variance is re- stricted to waves shorter than 3 to 6 times the peak wavelength, as proposed by Kerbaol (1997). At present we have not found a method to estimate this cut-off and thus recover wave heights right up to the ice edge using the images alone. As we have only used the amplitudes, it is possible that using the complex images may help reduce the speckle noise and better identify a spectral signature of the cut-off. However, this effect should be negligible at distances of $40 \mathrm{~km}$ or more from the ice edge and in the case of swells where the higher frequency components have already been attenuated in open water, outside the ice.

Going inside the ice, away from the edge, the imaging of waves by SAR becomes linear and should provide an accurate method for measuring wave heights. A verification of the method will require in situ data deeper inside the ice compared to the dataset used here. Also, we expect that in some images, such as Figure 10, it will be possible to determine floe sizes and thus investigate the relationship between wave damping and the floe sizes, once the ice thickness is 
known from measurements with other sensors.

Finally, a comparison of Sentinel 1 with TerraSAR-X, which flies at an altitude 30\% lower than Sentinel 1, will be very useful to further test the theoretical transformation of velocities into image patterns and the blurring effect by unresolved waves.

\section{Acknowledgement}

F.A. and J.S. are supported by Labex Mer via grant ANR-10-LABX-19-01, EU-FP7 project SWARP under grant agreement 607476, ONR grant number N0001416WX01117, and CNES, it is based on observations with Sentinel 1 kindly provided by ESA. The dedication of the crew of $\mathrm{R} / \mathrm{V}$ Sikuliaq was essential in gathering the in situ data. We also thank ESA and the Copernicus program for the exceptional quality of the SAR data, and making possible the acquisition of IW mode data over the Beaufort Sea. Discussions with and detailed comments from P. Sutherland helped improve the manuscript. Comments from the editor and three anonymous reviewers were very helpful for clarifying parts of this manuscript.

\section{References}

Alpers, W. R., Rufenach, C. L., 1979. The effect of orbital motions on synthetic aperture radar imagery of ocean waves. IEEE Trans. Antennas Propagat. 27 (C7), 685-690.

Ardhuin, F., Collard, F., Chapron, B., GirardArdhuin, F., Guitton, G., Mouche, A., Stopa, J., 2015. Estimates of ocean wave heights and attenuation in sea ice using the sar wave mode on Sentinel-1A. Geophys. Res. Lett. 42, 2317 2325 .
Ardhuin, F., Herbers, T. H. C., Watts, K. P., van Vledder, G. P., Jensen, R., Graber, H., 2007. Swell and slanting fetch effects on wind wave growth. J. Phys. Oceanogr. 37 (4), 908-931.

Ardhuin, F., Sutherland, P., Doble, M., Wadhams, P., 2016. Ocean waves across the Arctic: attenuation due to dissipation dominates over scattering for periods longer than $19 \mathrm{~s}$. Geophys. Res. Lett. 43, 5775-5783.

Chapron, B., Johnsen, H., Garello, R., 2001. Wave and wind retrieval from SAR images of the ocean. Ann. Telecommun. 56, 682-699.

Collard, F., Ardhuin, F., Chapron, B., 2005. Extraction of coastal ocean wave fields from SAR images. IEEE J. Oceanic Eng. 30 (3), 526-533.

Collard, F., Ardhuin, F., Chapron, B., 2009. Monitoring and analysis of ocean swell fields using a spaceborne SAR: a new method for routine observations. J. Geophys. Res. 114, C07023.

Collins, III, C. O., Rogers, W. E., Marchenko, A., Babanin, A. V., 2015. In situ measurements of an energetic wave event in the Arctic marginal ice zone. Geophys. Res. Lett. 42, 1863-1870.

Cox, C. S., 1958. Measurement of slopes of highfrequency wind waves. J. Mar. Res. 16 (3), 199-225.

Davis, P. E. D., Lique, C., Johnson, H. L., 2016. Competing effects of elevated vertical mixing and increased freshwater input on the stratification and sea ice cover in a changing arctic ocean. J. Phys. Oceanogr. 46, 1531-1553.

Doble, M. J., Carolis, G. D., Meylan, M. H., Bidlot, J.-R., Wadhams, P., 2015. Relating wave 
attenuation to pancake ice thickness, using field measurements and model results. Geophys. Res. Lett. 42, 4473-4481.

Engen, G., Johnsen, H., 1995. Sar-ocean wave inversion using image cross spectra. IEEE Trans. on Geosci. and Remote Sensing 33, 4.

Forget, P., Broche, P., Cuq, F., 1995. Principles of swell measurements by SAR with applications to ERS-1 observations off the Mauritanian coast. Int. J. Remote Sensing 16, 24032422 .

Hasselmann, K., Chapron, B., Aouf, L., Ardhuin, F., Collard, F., Engen, G., Hasselmann, S., Heimbach, P., Janssen, P., Johnsen, H., Krogstad, H., Lehner, S., Li, J.-G., Li, X.-M., Rosenthal, W., Schulz-Stellenfleth, J., 2012. The ERS SAR wave mode: a breakthrough in global ocean wave observations. In: ERS Missions: 20 Years of Observing Earth. European Space Agency, Noordwijk, The Netherlands, pp. 165-198.

Hasselmann, K., Hasselmann, S., 1991. On the nonlinear mapping of an ocean wave spectrum into a synthetic aperture radar image spectrum and its inversion. J. Geophys. Res. 96 (C6), 10713-10729.

Hasselmann, K., Raney, R. K., Plant, W. J., Alpers, W., Shuchman, R. A., Lyzenga, D. R., Rufenach, C. L., Tucker, M. J., 1985. Theory of Synthetic Aperture Radar ocean imaging: a MARSEN view. J. Geophys. Res. 90 (C3), 4659-4686.

Jähne, B., Riemer, K. S., 1990. Two-dimensional wave number spectra of small-scalewater surface waves. J. Geophys. Res. 95 (C7), 1153111546.
Johnsen, H., Collard, F., 2004. ASAR wave mode processing - validation of reprocessing upgrade. technical report for ESA-ESRIN under contract 17376/03/I-OL. Tech. Rep. 168, NORUT.

URL http://en.itek.norut.no/norut_ troms/publikasjoner/rapporter/asar wave_mode_validation_of_reprocessing_ upgrade

Johnsen, H., Collard, F., 2009. Sentinel-1 ocean swell wave spectra (OSW) algorithm definition. Tech. Rep. 13, NORUT.

URL https://sentinel.esa.int/c/ document_library/get_file?folderId= 349449\&name=DLFE-4504.pdf

Johnsen, H., Engen, G., Collard, F., Kerbaol, V., Chapron, B., 2006. Envisat ASAR wave mode products - quality assessment and algorithm upgrade. In: Proceedings of SEASAR 2006, SP-613. ESA, ESA - ESRIN, Frascati, Italy.

URL http://earth.esa.int/workshops/ seasar2006/proceedings/papers/s1_1_ joh.pdf

Kerbaol, V., 1997. Analyse spectrale et statistique vent-vagues des images radar ouverture synthtique : application aux donnes satellites ers-1/2. Ph.D. thesis, Université de Rennes 1.

Kerbaol, V., Chapron, B., Vachon, P., 1998. Analysis of ERS-1/2 synthetic aperture radar wave mode imagettes. J. Geophys. Res. 103 (C4), 7833-7846.

Kohout, A. L., Williams, M. J. M., Dean, S. M., Meylan, M. H., 2014. Storm-induced sea-ice breakup and the implications for ice extent. Nature 509, 604-607. 
Krogstad, H. E., 1992. A simple derivation of Hasselmann's nonlinear ocean-synthetic aperture radar transform. J. Geophys. Res. 97 (C2), 2421-2425.

Liu, A. K., Holt, B., Vachon, P. W., 1991. Wave propagation in the marginal ice zone' model predictions and comparisons with buoy and synthetic aperture radar data. J. Geophys. Res. 96 (C3), 4605-4621.

Longuet-Higgins, M. S., Cartwright, D. E., Smith, N. D., 1963. Observations of the directional spectrum of sea waves using the motions of a floating buoy. In: Ocean Wave Spectra, proceedings of a conference, Easton, Maryland. National Academy of Sciences, PrenticeHall, pp. 111-136.

Lygre, A., Krogstad, H. E., 1986. Maximum entropy estimation of the directional distribution in ocean wave spectra. J. Phys. Oceanogr. 16, $2,052-2,060$.

Lyzenga, D. R., Shuchman, R. A., Lyden, J. D., Rufenach, C. L., 1985. SAR imaging of waves in water and ice: Evidence for velocity bunching. J. Geophys. Res. 90, 1031-1036.

Pierson, Jr, W. J., Moskowitz, L., Dec. 1964. A proposed spectral form for fully developed wind seas based on the similarity theory of $\mathrm{S}$. A. Kitaigorodskii. J. Geophys. Res. 69 (24), 5,181-5,190.

Rascle, N., Ardhuin, F., 2009. Drift and mixing under the ocean surface revisited. stratified conditions and model-data comparisons. J. Geophys. Res. 114, C02016.

Shulz-Stellenfleth, J., Lehner, S., 2002. Spaceborne synthetic aperture radar observations of ocean waves traveling into sea ice. J. Geophys. Res. 107 (C8), 20-1-20-19.

Squire, V., Dugan, J., Wadhams, P., Rottier, P., Liu, A., 1995. Of ocean waves and sea ice. Annu. Rev. Fluid Mech. 27 (3), 115-168.

Stopa, J. E., Ardhuin, F., Chapron, B., Collard, F., 2015. Estimating wave orbital velocity through the azimuth cutoff from spaceborne satellite. J. Geophys. Res. 130, 76167634 .

Sutherland, P., Gascard, J. C., 2016. Airborne remote sensing of ocean wave directional wavenumber spectra in the marginal ice zone. Geophys. Res. Lett. 43, 4659-4664.

Thomson, J., 2012. Wave breaking dissipation observed with "swift" drifters. J. Atmos. Ocean Technol. 29, 1866-1882.

Vachon, P. W., Olsen, R. B., Krogstad, H. E., Liu, A. K., 1993. Airborne synthetic aperture radar observations and simulations for waves in ice. J. Geophys. Res. 98 (C9), 16411-16425.

Wadhams, P., Parmiggiani, F. F., de Carolis, G., Desiderio, D., Doble, M. J., 2004. SAR imaging of wave dispersion in Antarctic pancake ice and its use in measuring ice thickness. Geophys. Res. Lett. 31, L15305.

Wadhams, P., Squire, V. A., Ewing, J. A., Pascal, R. W., 1986. The effect of the marginal ice zone on the directional wave spectrum of the ocean. J. Phys. Oceanogr. 16, 358-376.

Wadhams, P., Thomson, J., 2015. The arctic ocean cruise of R/V Sikuliaq 2015. an investigation of waves and the advancing ice edge. Il Polo LXX-4, 9-38. 